\title{
Influences of Cognitive Structure Variables on Miners' Unsafe Behavior Intentions
}

\author{
Gonglong Shi, ${ }^{1,2}$ Haisong Tan, ${ }^{1,2}$ Hongxia Li ${ }^{10,2}$ Qian Wang, ${ }^{2,4}$ and Shuicheng Tian ${ }^{3,4}$ \\ ${ }^{1}$ School of Management, Xi'an University of Science and Technology, Xi'an 710054, China \\ ${ }^{2}$ Institute of Safety Management \& Risk Control, Institute of Safety \& Emergency Management, \\ Xi'an University of Science and Technology, Xi'an 710054, China \\ ${ }^{3}$ Graduate School, Xi'an University of Science and Technology, Xi'an 710054, China \\ ${ }^{4}$ College of Safety Science and Engineering, Xi'an University of Science and Technology, Xi'an 710054, China
}

Correspondence should be addressed to Hongxia Li; lihx@xust.edu.cn

Received 26 January 2021; Revised 19 February 2021; Accepted 16 March 2021; Published 31 March 2021

Academic Editor: Sang-Bing Tsai

Copyright ( 2021 Gonglong Shi et al. This is an open access article distributed under the Creative Commons Attribution License, which permits unrestricted use, distribution, and reproduction in any medium, provided the original work is properly cited.

In order to study the influence of cognitive structure variables on miners' unsafe behavior intentions, using TPB as the basic theoretical framework, a hypothesis model of miners' unsafe behavior intentions was constructed including three cognitive structure variables of risk perception, behavior experience, and safety awareness. The statistical results of 236 valid questionnaires were used to verify the authenticity of the model; the structural equation model was used to investigate and reveal the influence path of cognitive structure variables on miners' unsafe behavior intentions. This study shows that risk perception, behavior experience, and safety awareness have a significant positive impact on miners' unsafe behavior intentions. Behavior intentions have a guiding effect on behavioral outcomes. By adopting interventions, miners can be guided to reduce unsafe behaviors to avoid production safety accidents.

\section{Introduction}

In recent years, China has paid more and more attention to the management of safe production, and the work safety in the coal mining industry has been fruitful. In 2018, the mortality rate of one million tons of raw coal fell below 0.1 for the first time, which is the best level in the history of Chinese coal mining industry. But, compared with developed countries in the world, the gap is still obvious, and various types of coal mine accidents occur from time to time. In the first half of 2019 , more than 314,000 potential accidents were investigated and dealt with in coal mines nationwide, including 806 major hidden dangers. There were 73 fatal accidents and 131 deaths. When working underground in the coal mine, miners have to face complex operating environments, high labor intensity, and heavy psychological load, which easily breed all kinds of unintentional and unsafe behaviors. Statistics showed that more than $80 \%$ of coal mine accidents were caused directly or indirectly by miners' unsafe behaviors [1-3]. Therefore, strengthening the effective management and control of miners' unsafe behaviors is of great significance for preventing coal mine production safety accidents and ensuring safe production.

Intention is the tendency of individuals to respond to behavior objects. Behavior intention is the most obvious control variable to study the probability of behavior occurrence [4], which refers to individuals' subjective selfassessment of the degree of their own willingness or possibility to carry out a certain behavior in the future and the willingness to put efforts in the implementation of the planned behavior. In order to prevent the occurrence of unsafe behaviors of miners, it is necessary to understand the factors that lead to the implementation of unsafe behaviors of miners and to master the process of the development of intentions of miners' unsafe behaviors. As the most famous theory of relationship between attitude and behavior in social psychology, the Theory of Planned Behavior (TPB) [5] 
has been widely used to study human behavior intentions and behavior predictions. The TPB model is shown in Figure 1.

Based on the TPB as a basic framework, with the help of other intermediary variables, scholars at home and abroad have carried out relevant research on behavior intentions.

Li Nai-wen et al. [6] used the TPB as the theoretical framework to introduce two variables of past behaviors and exemplary norm and constructed a hypothesis model of deliberate violation behavior intention of miners. The study shows that these variables have a direct or indirect significant influence on deliberate violation behavior intentions of miners. Yang Jia-li et al. [3] built a model of influencing factors of unsafe behavior intention of miners on the basis of the TPB and carried out dynamic simulation of its action mechanism. It was found that adopting measures to optimize behavior attitude, subjective norm, and perceptual behavior control can effectively reduce the intensity of miners' unsafe behavior intentions. Wang Dan [7] introduced two variables of past behavior and risk tendency under the TPB framework. The results of SEM analysis showed that the introduced variables and the three major factors of the TPB all had an impact on the miners' violations. Tian Shui-cheng et al. [2] carried out research based on the TPB and found that factors such as attitude, subjective norm, perceptual behavior control, work stress, and risk tendency have a direct impact on unsafe behavior of miners. Asghar Bagheri et al. [8] found that ethical norm, subjective norm, and attitude had a significant impact on farmers' use of pesticides based on TPB research. Oliver [9], Bearden [10], and others had used empirical research to find that behavior attitude was related to subjective norm, that is, subjective norm had an impact on behavior attitude. The study by Bagozzi [11] also showed that behavior attitude was a mediating variable that behavior control influences behavior intention.

Most of the abovementioned studies have not studied the influencing factors of behavior intention from the perspective of cognition. Therefore, this paper will introduce three cognitive structure variables of risk perception, behavior experience, and safety awareness on the basis of the TPB to construct a model of the intention of unsafe behavior of miners and use SEM to reveal the influence of cognitive structure variables on the intention of unsafe behavior of miners. Intervention measures are proposed to effectively control the unsafe behavior of miners.

\section{Model Construction and Research Hypotheses}

\subsection{Cognitive Links Arising from Unsafe Behavior Intentions.} There are several modern popular cognitive models of behavioral psychology, such as the WICKENS information processing model [12], the RASMUSSEN decision process step model [13], the ATHEANA method [14], and the HOLLNAGEL context-dependent control model [15], which consider behavioral cognition. The process of behavioral cognition included four stages: perception of external information, judgment and analysis of information, choice of

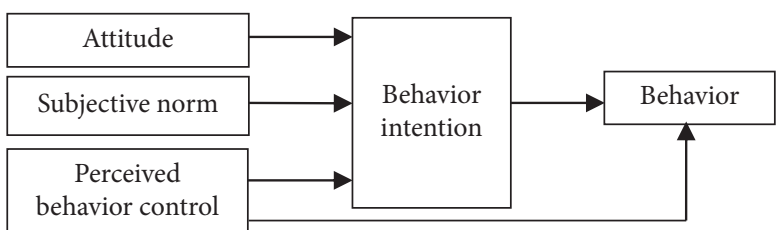

Figure 1: Model of the TPB.

response behavior, and output of behavior, which can be simplified into finding information, understanding information, selecting response, and implementing response, as shown in Figure 2.

However, many empirical studies have found that when people are aware of the surrounding environment and feeling the imminent danger, they will first judge the nature of the danger based on existing information such as previous experience, then think about how to deal with the danger, choose the best response measures, and finally, implement the selected measures to deal with the danger. Therefore, the process of generating a more complete behavioral cognition should include at least five links: discovering information, understanding information, thinking and coping, choosing coping, and implementing coping. So, the structure of the behavioral cognitive links adopted in this paper is as shown in Figure 3.

The behavior is controlled by intention, and the unsafe behavior intention of the miners will cause its unsafe behavior to occur. Therefore, to study the behavior intentions of miners before the implementation of unsafe behaviors, we should start with the analysis of the information discovery, understanding, thinking, and response in the process of behavior cognition [16].

2.2. Construction of the Basic Model. Risk perception is a subjective judgment made by people about the characteristics and severity of a particular risk. Miners' perception and understanding of dangerous scenarios is the judgment of the probability of possible dangers in the operating environment and the severity of their consequences, and the degree of perception of this dangerous scenario depends on the degree of discovery of the dangerous information in the environment by the miners.

Safety awareness is people's vigilance and alertness to various surrounding environmental conditions that may cause harm to themselves or others during production activities. In this state of mind, the miners give different meanings to various types of hazard information in the surrounding environment and evaluate the possible consequences and impact of the hazard if it occurs.

Behavior experience is the experience and lessons people learn by summarizing and reflecting on past life and work experiences and using it to compare and judge the abnormal situation in the work environment. When the environment changes, the miners will judge whether there is a danger based on the scenes in the memory and use their past experience to determine the appropriate risk avoidance measures. 


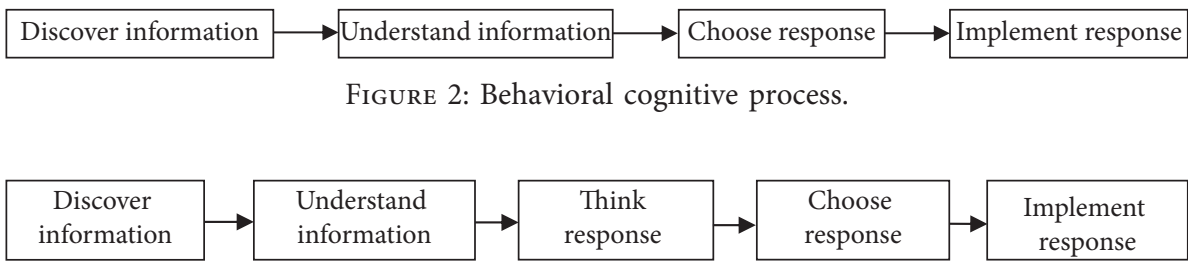

FIGURE 3: Five-link composition model of the behavioral cognitive process.

Behavior attitude is the subjective value evaluation that people have for a certain behavior. In the process of operation, miners have relatively stable views on the behavior of others and themselves, and this solid view will have a certain impact on the behavior of miners.

Subjective norm is the social pressures that people may feel for taking a certain behavior. In general, when people intend to take a certain behavior, they will consider the public views on this behavior.

Perceptual behavior control is the speculation and judgment made by an individual as to whether he or she is capable of performing a certain behavior, and it is a selfevaluation of whether a person has a certain behavioral ability.

Parker [17] believed that the social psychology reasons for the failure of the selection response could be analyzed from the three factors of behavior attitude, subjective norm, and perceptual behavior control, and these three factors are exactly the three core components of the PBT [18].

2.3. Assumption Model of Miners' Unsafe Behavior. Scientific research has shown that human behavior is the product of cognition, safe behavior is generated by correct cognition, and unsafe behavior is caused by cognitive failure. Therefore, the failure of the cognitive link will inevitably lead to the unsafe behavior intention of miners. We can make the following assumptions:

$\mathrm{H} 1$ : risk perception positively affects the intention of unsafe behavior of miners

$\mathrm{H} 2$ : safety awareness positively affects the intention of unsafe behavior of miners

H3: behavior experience positively affects the intention of unsafe behavior of miners

$\mathrm{H} 4$ : behavior attitude positively affects the intention of unsafe behavior of miners

H4: subjective norm positively affects the intention of unsafe behavior of miners

H6: perceptual behavior control positively affects the intention of unsafe behavior of miners

According to the successive relationship of each link in the behavior cognition process, it is reasonable to infer that risk perception positively affects safety awareness and safety awareness positively influences behavior experience, and the miner's behavior attitude will be affected by risk perception, safety awareness, and behavior experience. Based on this, the following assumptions are made:
H7: risk perception positively affects the miners' unsafe behavior intentions by safety awareness

H8: safety awareness positively affects the miners' unsafe behavior intention by behavior experience

H9: risk perception positively affects miners' unsafe behavior intentions by behavioral attitude

H10: safety consciousness positively affects the miners' unsafe behavior intentions by behavior attitudes

H11: behavioral experience positively affects miners' unsafe behavior intentions by behavioral attitudes

H12: subjective norms positively affect miners' unsafe behavior intentions by behavioral attitudes

H13: perceptual behavior control positively affects miners' unsafe behavior intentions by behavior attitudes

Based on the TPB, combined with the cognitive process of miners' behavior, with the help of three cognitive structural variables of risk perception, safety awareness, and behavior experience, a model of miners' unsafe behavior intention is constructed, as shown in Figure 4.

\section{Research Methods}

3.1. Research Object. The target samples were obtained from the coal mining driver, hydraulic support worker, and scraper driver of the fully mechanized underground mining operations, and they are from Shenmu Hongliulin Mining Co., Ltd. of Shaanxi Coal Industry Company Limited and Daliuta Coal Mine of China Shenhua Energy Company Limited. The questionnaire survey was carried out by two methods: on site and online. A total of 318 questionnaires were recovered within the agreed time. Invalid questionnaires such as incomplete information and missing options were excluded. A total of 236 valid questionnaires were obtained, with an effective rate of $74.21 \%$, which met the requirements of SEM statistical analysis. The basic statistics of individual miners participating in the questionnaire survey are shown in Figure 5.

3.2. Scale Design. With reference to Shanxi Coal Transportation and Marketing Group Yangcheng Huiyang Coal Industry Co, Ltd. "Code for Operation of Comprehensive Mining Operators," the measurement items of relevant variables were scored using the LIKERT five-component scale method, and a preliminary draft of the questionnaire was designed using the standardized direct measurement method. By on-site surveys and visits by miners and 


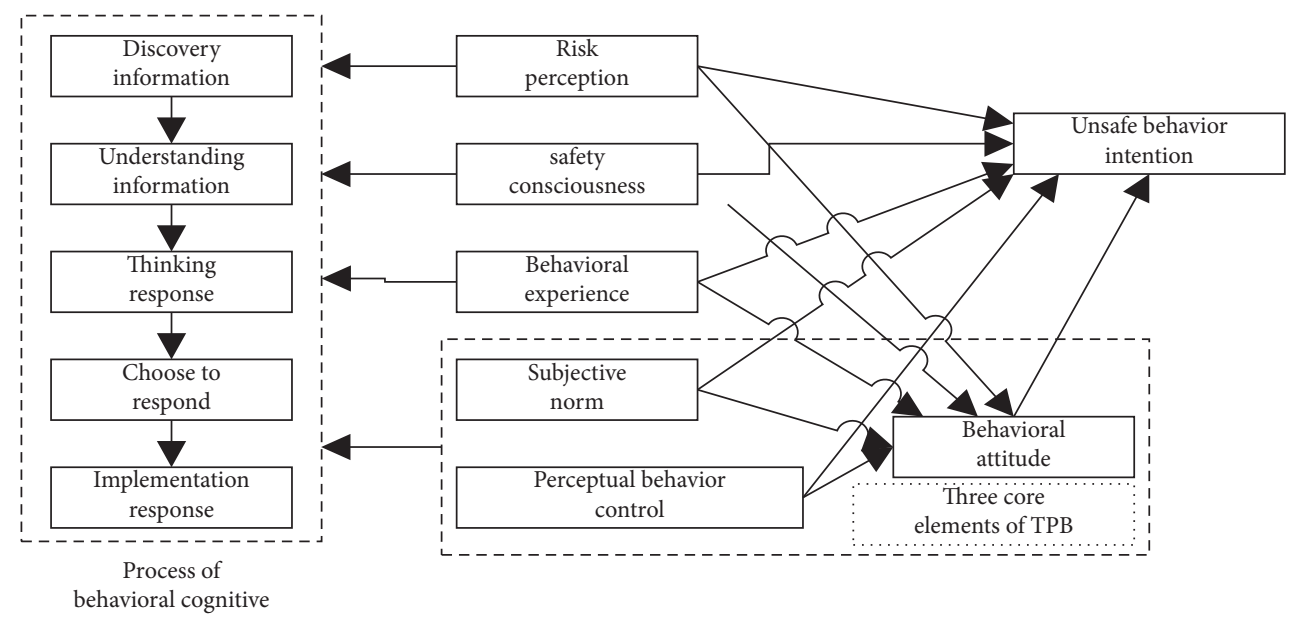

FIgURE 4: Model for miners'unsafe behavior intention.
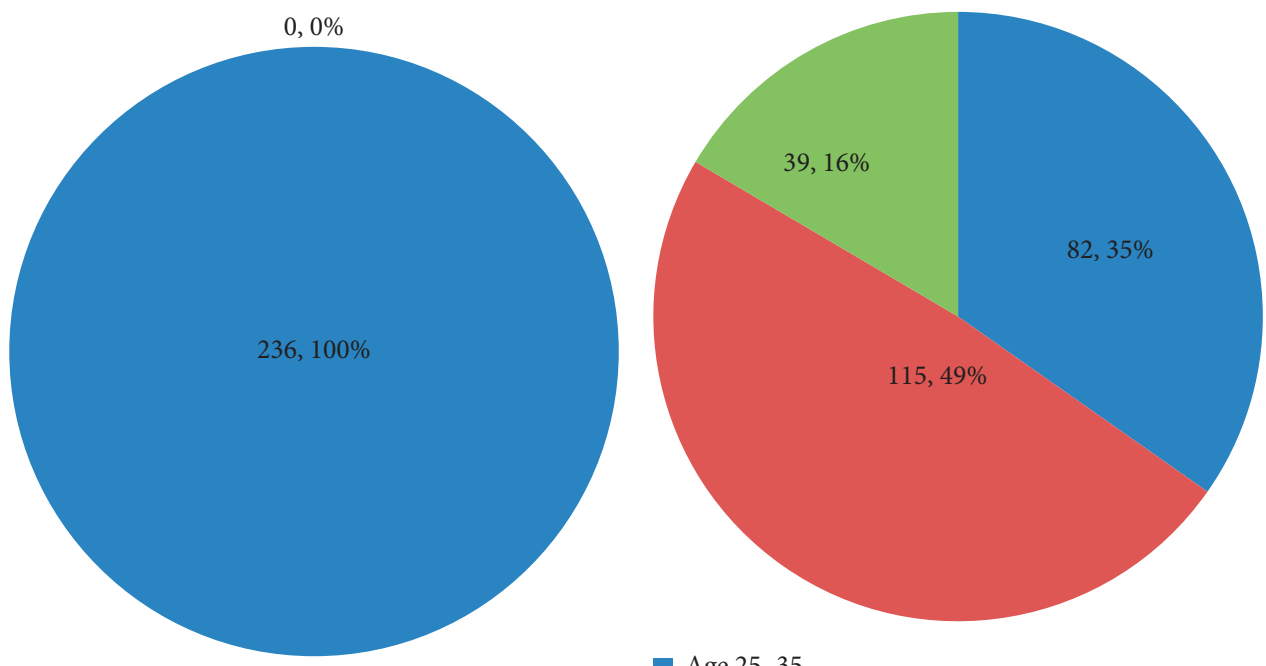

Gender male

Age 25-35

Gender female

Age 35-45

Age 45-55

(a)

(b)

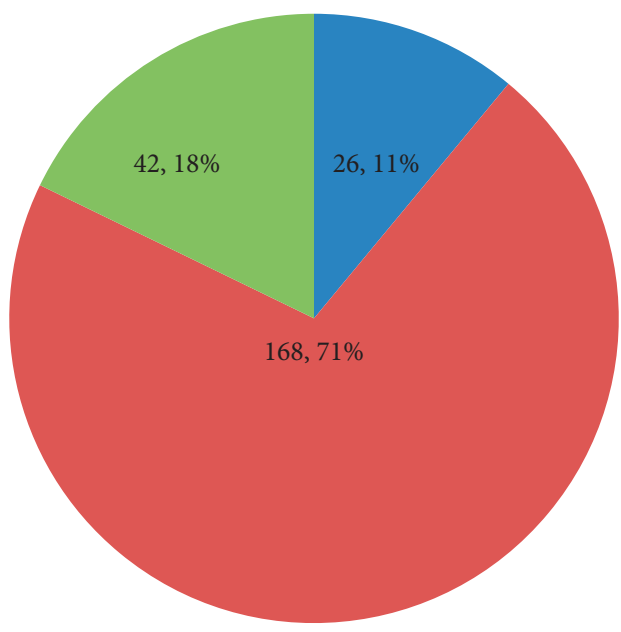

Marriage unmarried

Marriage married

Marriage divorced

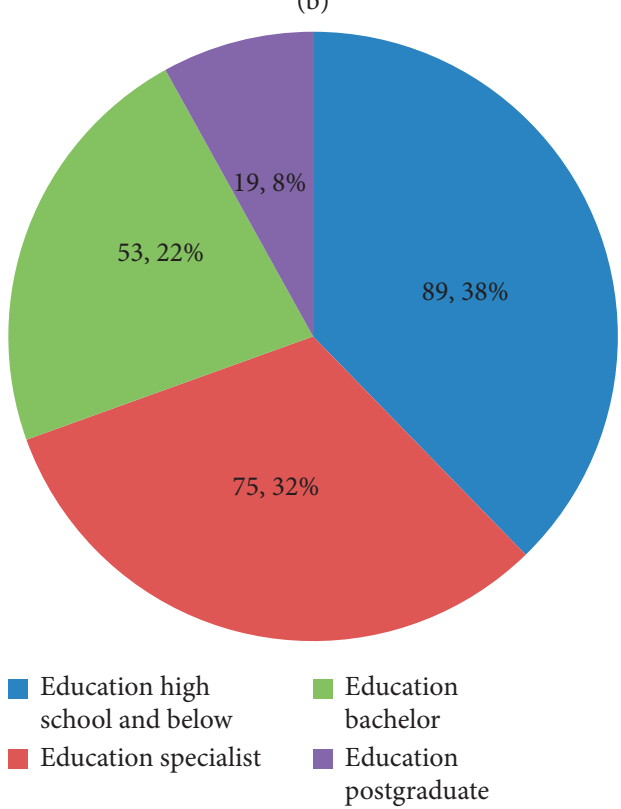

(c)

(d)

FIgURE 5: Basic situation of miners. 
appropriate adjustments to the items in combination with the characteristics of fully mechanized coal mining operations in the coal mines have resulted in a revised version of the questionnaire on unsafe behavior intentions of miners. It verified the strength of the relationship between the latent variables and the intention of unsafe behavior: the latent variable score indicates the strength of the corresponding behavior ability; the unsafe behavior intention score indicates the possibility of the miner's tendency to adopt some unsafe behavior.

The questionnaire was designed by three questions for each variable. Among them, items such as "I think the risk of fully mechanized mining operations is high" were used to measure risk perception; items such as "I will be more careful in operation in hazardous areas" were used to measure safety awareness; "I have had unsafe behaviors in the past, but no accidents and no punishments" was used to measure behavior experience; "In order to complete the work as quickly as possible, I have shortcuts, and do not follow procedures" and other items were used to measure behavior attitude; in order to measure subjective norm, items such as "Everyone is strongly opposed to someone moving under the roof hydraulic support or in front of them" was been used; "I think safe behavior is difficult to achieve" was used to measure perceptual behavior control; items such as "I think operating procedures are too cumbersome, and I may choose to take shortcuts" and others were used to measure the intention of unsafe behavior.

\section{Data Analysis and Result Discussion}

SPSS22.0 and AMOS22.0 were used for statistical analysis of the questionnaire data, and the hypothesis relationship was tested by SEM.

4.1. Reliability and Validity Test. Cronbach's $\alpha$ coefficient was used to test the internal consistency of the questionnaire data. Cronbach's $\alpha$ coefficient of each variable was greater than 0.8 , indicating that the reliability of the questionnaire was high, and the result is shown in Table 1.

The KMO score of the questionnaire was 0.836 , and the Bartlett's Spherical Test Sig $=0.000$, which can be used for factor analysis. The factor load of all measured indicators on their respective latent variables was from 0.673 to 0.894 , and this showed that the validity of the questionnaire was high.

4.2. SEM Fitted Test. The hypothesis model of miners' unsafe behavior intention was fitted, and the result is shown in Table 2.

The result in Table 2 showed that the model fitted well. Therefore, the relationship model of the influencing factors of the miners' unsafe behavior intention can be determined, as shown in Figure 6.

Among them, safety awareness, behavior experience, and behavior attitude $(\beta=0.219, p<0.001 ; \beta=0.199, p<0.001$; and $\beta=0.257, p<0.001$ ) all significantly affect the intention of unsafe behavior; subjective norm and perceptual behavior control $(\beta=0.114, p<0.05 ; \beta=0.133, p<0.05)$ significantly affect the intention of unsafe behavior. Risk perception $(\beta=0.485, p<0.001)$ significantly indirectly positively influences the intention of unsafe behavior by the mediating variable of safety awareness; safety awareness $(\beta=0.361$, $p<0.001)$ indirectly positively influences the intention of unsafe behavior by the intermediary variable of behavior experience; risk perception, safety awareness, behavioral experience, subjective norm, and perceptual behavior control $(\beta=0.456, \quad p<0.001 ; \beta=0.133, \quad p<0.01 ; \beta=0.199$, $p<0.001 ; \beta=0.199, p<0.001$; and $\beta=0.209, p<0.001)$ indirectly positively and significantly affect unsafe behavior intention by the mediating variable of behavior attitude. Obviously, all hypotheses had been verified except H1.

4.3. Result Discussion and Management Measure. As an important intermediary variable, behavior attitude is the most directly significant among all latent variables that affect the intention of unsafe behavior of miners. Coal enterprises would strengthen the education of miners' safety attitude and guide them to establish a correct outlook on safety; miners also would communicate more with colleagues, friends, and family members, reasonably release their psychological depression, straighten their attitude, and reduce unwarranted complaint.

Risk perception has the greatest impact on miners' unsafe behavior intention by safety awareness, and it also significantly affects unsafe behavior intention by behavior attitude. In daily management work, coal enterprises can improve the level of miners' risk perception with education and training and use the risk management information system to improve the miner's ability of risk identification and enhance risk awareness. In terms of education, it can be introduced through the personal experience of the accident, the VR technology-based experiential training of accident reconstruction, the formation of safety psychology, and other popular ways, so that miners can deeply and truly understand and be alert to the seriousness of the consequences of unsafe behavior, so as to maintain a high level of risk perception.

Safety awareness and behavior experience also have a significant influence and positive correlation on unsafe behavior intention. Through the promotion of safety laws and regulations, the implementation of standardized operations, and the implementation of cyclical safety system education, the ability of miners' to accurately identify risks, the ability to respond to decisions in a scientific and rational manner, and enhance the awareness of risk prevention can be increased; the method of "finger dictation" can be adopted to cultivate miner safety ability to work, develop strong incentives to curb unsafe behavior, encourage safe behavior, and strengthen guidance for safe behavior, and all kinds of measures should be taken to ensure the safety of everything, all the time and everyone.

Perceptual behavior control and subjective norm, which are the core elements of the TPB, have a significant effect on the intention of unsafe behavior of miners. The ways, family members picking up miners from mine wells and colleagues reminding each other kindly, can be adopted to affect and 
TABle 1: Cronbach's $\alpha$ of each variable.

\begin{tabular}{|c|c|c|c|c|c|c|c|}
\hline Variable & $\begin{array}{c}\text { Risk } \\
\text { perception }\end{array}$ & $\begin{array}{c}\text { Safety } \\
\text { awareness }\end{array}$ & $\begin{array}{l}\text { Behavior } \\
\text { experience }\end{array}$ & $\begin{array}{l}\text { Behavior } \\
\text { attitude }\end{array}$ & $\begin{array}{c}\text { Subjective } \\
\text { norm }\end{array}$ & $\begin{array}{c}\text { Perceptual } \\
\text { behavior control }\end{array}$ & $\begin{array}{c}\text { Unsafe behavior } \\
\text { intention }\end{array}$ \\
\hline $\begin{array}{l}\text { Cronbach's } \alpha \\
\text { coefficient }\end{array}$ & 0.913 & 0.850 & 0.906 & 0.861 & 0.819 & 0.851 & 0.902 \\
\hline
\end{tabular}

TABLE 2: Fit indexes of the model.

\begin{tabular}{cccccccc}
\hline $\mathrm{X} 2 / \mathrm{df}$ & CFI & GFI & AGFI & IFI & NFI & TLI & RMSEA \\
\hline 2.322 & 0.906 & 0.830 & 0.789 & 0.912 & 0.904 & 0.897 & 0.071 \\
\hline
\end{tabular}

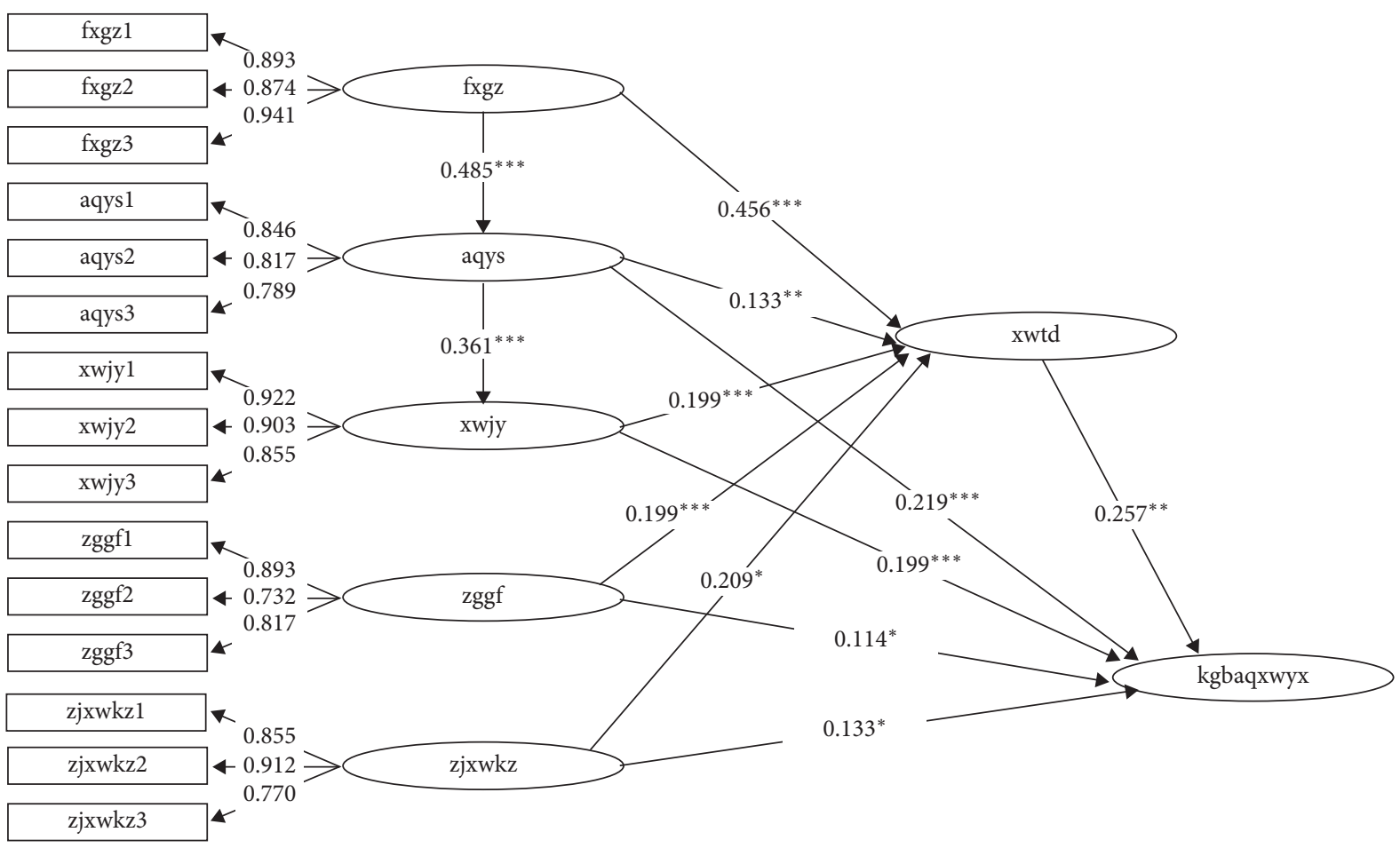

Note: ${ }^{*}$ means $p<0.05,{ }^{* *}$ means $p<0.01 ;{ }^{* * *}$ means $p<0.001$.

FIGURE 6: Relational model of influence factors for miners' unsafe behavior intention.

strengthen the safety awareness of miners, and a safe working atmosphere will be built, which is of mutual help and friendly; a comprehensive coal monitoring and inspection system can be established to continuously monitor the safety status of miners by the use of advanced psychophysiological information collection technology; the exemplary leadership role of the team leaders in compliance with the rules and discipline should be brought into full play, and the supervision enthusiasm of the employees could be improved; in order to give full play to the positive guiding role of subjective norm and behavior experience to regulate and restrict the behavior of miners, strong incentives and penalties could be implemented. Coal enterprises should guarantee the procurement and use of intrinsically safe advanced production equipment from the capital input, ensure adequate and timely supply of safety protective equipment from the system, and strive to avoid various triggers of unsafe behavior of miners.

\section{Conclusions}

5.1. The Validity of the Miner's Unsafe Behavior Intention Model Has Been Further Verified. With the help of the basic cognitive structure variables of the TPB, by introducing three new cognitive structure variables of risk perception, behavior experience, and safety awareness, a hypothesis model of miners' unsafe behavior intention was established. The result of data analysis showed that the risk perception of the mediator variable safety awareness positively affects the intention of unsafe behavior of miners. The effect of "discovery information" on "understanding information" in the behavior cognition link has been initially verified; the 
mediating variable of behavior experience has a positive and significant impact on the intention of miners' unsafe behavior. Thus, the effect of "understanding information" on "thinking and coping" in behavioral cognition has also been verified. By the mediator variable of behavioral attitude, risk perception, safety awareness, and behavioral experience all significantly positively affect unsafe behavior intentions. It can be seen that the abovementioned three links of behavior cognition also have a significant impact on the link of "choose response." Therefore, the validity of the model of miners' unsafe behavioral intention, based on the cognitive process of behavior, has been further verified.

\subsection{The TPB Basic Model and the Three Cognitive Structural} Variables Introduced Have Been Verified. As the core elements of the TPB, the three latent variables of behavior attitude, subjective norm, and perceptual behavior control all have a significant positive impact on the intention of unsafe behavior of miners. Therefore, the basic model of the TPB has also been verified, and the three latent variables introduced as risk perception, safety awareness, and behavior experience also have a direct or indirect positive impact on miners' unsafe behavior intention, which indicates that the three latent variables are also suitable as the introduction variables of the TPB. It can be seen that it is feasible to analyze the influence of cognitive structural variables on the intention of miners' unsafe behavior.

5.3. Assumptions for Further Research. The result of research shows that the influence of the three major elements of TPB (behavior attitude, subjective norm, and perceptual behavior control) and the newly introduced three cognitive structural variables (risk perception, behavior experience, and safety awareness) on the unsafe behavior intention of miners has been tested. However, the influence of these variables on the implementation of unsafe behavior of miners has not been further explored, and the influence relationship between unsafe behavior intention and unsafe behavior implementation has not been analyzed; in other words, the influence of cognitive structure variables on the "implement response" in the process of behavior cognition has not been explored. Of course, there may be other influencing factors from intention to behavior, which can be taken as the direction of further research in the later stage.

\section{Data Availability}

The data that support the findings of this study are available from the corresponding author upon reasonable request.

\section{Conflicts of Interest}

The authors declare that they have no conflicts of interest.

\section{Acknowledgments}

This work was supported by the National Natural Science Foundation of China (Recognition and Simulation Research of High Probability Near-Miss by Multi-Source information Fusion and Hesitancy Fuzzy Decision-making, 51874237; Research on risk identification of concealed disaster-causing factors, early warning and emergency management of coal mine major disaster, U1904210; Research on resource integration mechanism of major emergencies psychological crisis intervention based on multiple collaborative perspectives, 20XGL025); the National Humanities and Social Science Project (17BJR01132); Research Fund Project From Xi'an University of Science and Technology (201617 and 2017SY07); and the Industry-academic cooperation collaborative education project from Ministry of Education of China (201702038141, 201801198016, and 202002261016).

\section{References}

[1] L. Li, S. C. Tian, and J. Deng, "Study on the coal miners' unsafe behavior: effective factors analysis and control measures," Journal of Xi'an University of Science and Technology, vol. 31, no. 6, pp. 794-813, 2011.

[2] T. S. Cheng, F. Liu, Y. Lu et al., "Study on miners' unsafe behaviors based on theory of planned behavior," Mining Safety \& Environmental Protection, vol. 41, no. 01, pp. 109$112,2014$.

[3] Y. Jia-Li, L. I. Ji-Zu, F. Guo-Rui et al., "Simulation research on factors influencing miners' unsafe behavioral intention and its application," China Safety Science Journal, vol. 26, no. 7, pp. 46-51, 2016.

[4] I. Ajzen, "The theory of planned behavior," Organizational Behavior and Human Decision Processes, vol. 50, no. 2, pp. 179-211, 1991.

[5] I. Ajzen, "From intentions to actions: a theory of planned behavior," in Action Control: From Cognition to Behavior, J. Kuhl and J. F. Beckman, Eds., Springer, Heidelberg, Germany, 1985.

[6] L. I. Nai-Wen, M. A. Yue, and N. I. U. Li-Xia, "Research on miners' deliberate violation behavior intentions based on theory of planned behavior," China Safety Science Journal, vol. 21, no. 10, pp. 3-9, 2011.

[7] D. Wang, "Study of miners' violation behaviors based on theory of planned behavior," China Safety Science Journal, vol. 21, no. 4, pp. 7-12, 2011.

[8] A. Bagheri, A. Bondori, M. Sadegh Allahyari et al., "Modeling farmers' intention to use pesticides: an expanded version of the theory of planned behavior," Journal of Environment Management, vol. 248, no. 10, 2019.

[9] A. Oliver, A. Cheyne, and J. M. Tomás, "The effects of organizational and individual factors on occupational accidents," Journal of Occupational and Organizational Psychology, vol. 75, no. 4, pp. 473-488, 2002.

[10] Y.-Y. Cox and X. Zhao, "Research on recycling behavior of waste household appliances and electronics based on TPB: a case of Shanghai," Management Review, vol. 21, no. 8, pp. 85-94, 2009.

[11] R. P. Bagozzi, K.-H. Lee, and M. F. Van Loo, "Decisions to donate bone marrow: the role of attitudes and subjective norms across cultures," Psychology \& Health, vol. 16, no. 1, pp. 29-56, 2001.

[12] C. D. Wickens, Engineering Psychology and Human Performance, Harper Collins Publishers, New York, NY, USA, 1992.

[13] J. Rasmussen, "Human errors. A taxonomy for describing human malfunction in industrial installations," Journal of Occupational Accidents, vol. 4, no. 2-4, pp. 311-333, 1982. 
[14] S. E. Cooper, J. Wreathall, C. M. D. M. Thompson et al., "Knowledge-base for the new human reliability analysis method, a technique for human error analysis (ATHEANA)," Office of Scientific \& Technical Information Technical Reports, vol. 84, no. 4, pp. 477-485, 1996.

[15] E. Hollnagel, Cognitive Reliability and Error Analysis Method (CREAM), pp. 262-275, Elsevier Science, London, UK, 1998.

[16] J. I. N. Liang-Hai, R. Gong, S. Chen et al., "Influences of cognitive structure variables onlifting workers' intentions to violate," China Safety Science Journal, vol. 28, no. 1, pp. 8-13, 2018.

[17] J. F. Parker, "A human factors guide for aviation maintenance," Proceedings of the Human Factors and Ergonomics Society Annual Meeting, vol. 37, no. 1, pp. 30-33, 1993.

[18] W.-T. Duan and G.-R. Jiang, "A review of the theory of planned behavior," Advances in Psychological Science, vol. 2, pp. 315-320, 2008. 